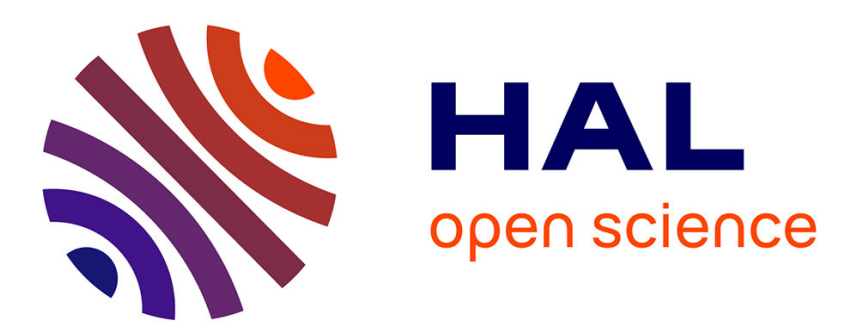

\title{
Phase-based order separation for Volterra series identification
}

Damien Bouvier, Thomas Hélie, David Roze

\section{To cite this version:}

Damien Bouvier, Thomas Hélie, David Roze. Phase-based order separation for Volterra series identification. International Journal of Control, 2019, pp.1-11. 10.1080/00207179.2019.1694175 . hal02920505

\section{HAL Id: hal-02920505 \\ https://hal.science/hal-02920505}

Submitted on 2 Sep 2020

HAL is a multi-disciplinary open access archive for the deposit and dissemination of scientific research documents, whether they are published or not. The documents may come from teaching and research institutions in France or abroad, or from public or private research centers.
L'archive ouverte pluridisciplinaire HAL, est destinée au dépôt et à la diffusion de documents scientifiques de niveau recherche, publiés ou non, émanant des établissements d'enseignement et de recherche français ou étrangers, des laboratoires publics ou privés. 


\section{Phase-based order separation for Volterra series identification}

Damien Bouvier ${ }^{\mathrm{a}}$, Thomas Hélie ${ }^{\mathrm{a}}$ and David Roze

a Team Sound Signals and Systems : Audio/Acoustics, instruMents (S3AM), Sorbonne Université, Ircam, CNRS, Ministère de la Culture, STMS, 1, Place Igor Stravinsky, F-75004 Paris, France

\section{ARTICLE HISTORY}

Compiled September 2, 2020

The Version of Record of this manuscript has been published and is available in International Journal of Control, 25 november 2019 (https : / www . tandfonline.com/). https://dx.doi.org/10.1080/00207179.2019.1694175 


\begin{abstract}
This article addresses identification of nonlinear systems represented by Volterra series. To improve the robustness of some existing methods, we propose a pre-processing stage that separates nonlinear homogeneous order contributions from which Volterra kernels can be identified independently. Unlike existing separation methods that use amplitude relations between test signals, we propose another order separation method based on phase. This method gives access to a new type of complex-valued output signals, which can be used to improve kernel identification.

First, the underlying ideas are introduced via the presentation of a theoretical method using complex-valued test signals. Second the proposed order separation method using real-valued signals is described. Third, a new identification process is given, combining existing leastsquares identification method with the previous results. Finally, numerical experiments are used to compare the proposed order separation method with state-of-the art, as well as to evaluate the new Volterra series identification process.
\end{abstract}

\title{
KEYWORDS
}

System identification: Nonlinear systems: Volterra series: Least-squares identification 


\section{Introduction}

Nonlinear system identification is a very challenging topic. One approach, using Volterra series (Volterra, 1887), has begun in the 40's with the seminal work of Wiener (1942). One characteristic that led to the widespread use of Volterra series is the large class of models that they can represent (Boyd \& Chua, 1985). But this advantage comes with important difficulties concerning identification, such as:

- the high number of kernels coefficients to estimate, which increases drastically with respect to the truncation order and the kernels memory; this leads to high variance on the estimated parameters.

- the simultaneous estimation of all kernels from the direct output (as shown in Fig. 1a); indeed, for a system that allows a Volterra series representation, amplitudes generally decreases as the order $n$ increases, thus making estimation of the higher-order kernels less reliable.

The first problem can be alleviated in different ways. Tensor decomposition (Batselier, Chen, \& Wong, 2017; Favier, Kibangou, \& Bouilloc, 2012), polynomial kernel regression (Franz \& Schölkopf, 2006) or projections on orthogonal basis, such as Laguerre (Marmarelis, 1993), Kautz (da Rosa, Campello, \& Amaral, 2007) or Generalized Orthogonal Basis (Kibangou, Favier, \& Hassani, 2005), greatly reduces the number of parameters to estimate, and therefore the overall complexity. The system can also be approximated using a block structure (Schoukens \& Tiels, 2017) , e.g. Hammerstein, Wiener or Wiener-Hammerstein structure, where the system is decomposed with only linear filters and static nonlinearities; for specific structures, estimation methods with good results are available (Rébillat, Hennequin, Corteel, \& Katz, 2011; Tiels \& Schoukens, 2014). Alternatively, regularization methods can be used to improve kernels estimation (Birpoutsoukis, Csurcsia, \& Schoukens, 2018; Birpoutsoukis, Marconato, Lataire, \& Schoukens, 2017).

The second problem is more difficult to circumvent. One approach is to divide the identification into 2 steps (as shown in Fig. 1b):

1. Order separation: nonlinear homogeneous orders $y_{n}$ are separated from a set of output signals;

2. Kernel identification: Volterra kernels $h_{n}$ are identified separately from each signal $y_{n}$.

This two-step approach decreases computational cost and increases robustness of the overall kernels estimation by splitting the identification process into $N$ smaller and independent

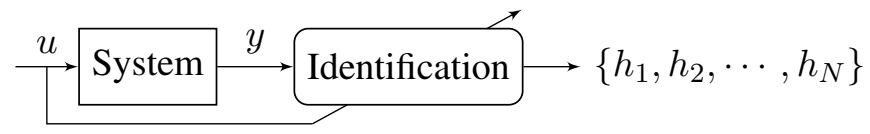

(a) Direct identification

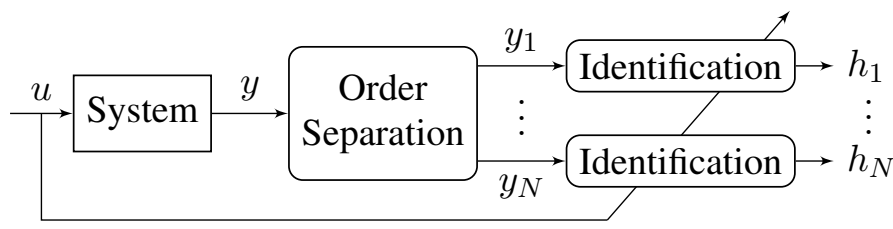

(b) Identification on separated order

Figure 1. Identification process of kernels $\left\{h_{n}\right\}_{n=1, \ldots, N}$ without (a) and with (b) prior order separation 
problems. Furthermore, this procedure is modular, meaning that any existing identification methods can be used in step 2. But it has to be noted that, due to the fact that order separation methods require specific user-defined input, this approach can not be used with any set of measurements (e.g. pre-recorded datasets).

In the literature, order separation is processed by using a collection of amplitude gains applied to a common input signal (Halme, Orava, \& Blomberg, 1971; Simpson \& Power, 1972); it has been used for identification purposes in e.g. Bard and Sandberg (2007); Boyd, Tang, and Chua (1983); Lee (1997); Zhang and Billings (2017). But this method relies on the inversion of a real-valued Vandermonde matrix, which is ill-conditioned when its size grows (Pan, 2016). In practice, this method requires numerous amplitude gains which have to be carefully chosen in order to provide good separation.

Recently, a separation method using phase deviations and amplitude gains has been proposed (Bouvier, Hélie, \& Roze, 2017), for which previous remarks on conditioning of the Vandermonde matrices still apply. Those ideas have been further developed in Bouvier, Hélie, and Roze (2018) leading to another kind of separation into homophase signals, which has been used to develop new identification processes. The use of phase deviations can be related to the results of Gardner and Archer (1993), where, in a probabilistic framework, cyclostationary signals are used to obtain orthogonality between orders ${ }^{1}$ (in a cross-correlation sense).

But even with those new developments, robust order separation can not yet been achieved: the method of Bouvier et al. (2017) still uses a set of amplitude gains leading to conditioning problems, and the method proposed in Bouvier et al. (2018) does not separate homogenous orders. This paper presents a new parameter-free order separation method exclusively based on phase deviation between excitation signals. This separation depends on the inversion of a 2D Discrete Fourier Transform and sparse matrices. Its contribution to Volterra series identification is illustrated with the introduction of a new Least-Squares identification method. All presented algorithms are available in an open-source Python toolbox ${ }^{2}$.

This paper is organized as follows: in Section 2, we give some mathematical foundations for the Volterra series paradigm and recall the existing separation method. Section 3 recalls basics on the phase-based order separation for the abstract case of complex-valued inputoutput signals; then, its robustness properties to noise and truncation error are analysed. Section 4 presents the main result, that is, an adaptation of the separation method to real-valued input-output signals to be used on physical systems. From this result, Section 5 derives a new Volterra series identification method. Finally, evaluation and comparison between several methods are made in Section 6, before giving some conclusions and perspectives.

\section{Volterra series and order separation}

\subsection{Notations and functional setting}

Introduce the following notations and definitions (with $n \in \mathbb{N}^{*}$ where $\mathbb{N}^{*}=\mathbb{N} \backslash\{0\}$ ):

- $L^{p}(\mathbb{X}, \mathbb{Y})$ : standard Lebesgue-space of $p$-normed functions from vector spaces $\mathbb{X}$ to $\mathbb{Y}$;

- $\mathbb{T}$ : set of times $\mathbb{R}, \mathbb{R}_{+}$or $[0, T]$ with $T>0$;

- $\mathcal{U}=\mathcal{Y}:=L^{\infty}(\mathbb{T}, \mathbb{R})$ : sets of input and output signals;

- $\mathcal{V S}$ : set of series $\left\{h_{n}\right\}_{n \in \mathbb{N}^{*}}$ with $h_{n} \in L^{1}\left(\mathbb{T}^{n}, \mathbb{R}\right)$ such that

○ $\rho_{h}>0$ is a non zero convergence radius of

\footnotetext{
${ }^{1}$ The links between the notion of order separation and order orthogonality (which is the key basis of the Wiener series framework) are not addressed here and deserve further study; for identification methods using orthogonalization approaches, see (Kibangou \& Favier, 2010; Tseng \& Powers, 1995).

${ }^{2}$ Available at https://github.com/d-bouvier/pyvi
} 
$\circ \phi_{h}: x \mapsto \sum_{n \in \mathbb{N}^{*}}\left\|h_{n}\right\|_{1} x^{n}$, the gain bound function;

- $\mathcal{V}_{n}:=\mathcal{M L}_{n}(\mathcal{U}, \mathcal{Y})$ : set of bounded (continuous) $n$-linear operators $\mathrm{V}_{n}: \mathcal{U}^{n} \rightarrow \mathcal{Y}$.

\subsection{Volterra series}

This section presents some recalls on Volterra series for bounded-input bounded-output (BIBO) systems. More details can be found in e.g. Boyd, Chua, and Desoer (1984); Rugh (1981).

Definition 2.1 (Volterra series). A BIBO causal time-invariant system is described around its equilibrium $y_{0} \in \mathbb{R}$ by a Volterra series $\left\{h_{n}\right\}_{n \in \mathbb{N}^{*}} \in \mathcal{V} \mathcal{S}$ if, for all inputs $u \in \mathcal{U}$ such that $\|u\|_{\infty}<\rho_{h}$, its output $y$ is given by, for all $t \in \mathbb{T}$,

$$
y(t)=\mathrm{V}[u](t):=\underbrace{\mathrm{V}_{0}}_{=y_{0}}+\sum_{n=1}^{\infty} \underbrace{\mathrm{V}_{n}[u, \ldots, u](t)}_{=y_{n}(t)},
$$

with $\mathrm{V}_{0}$ the constant operator of value $y_{0}$, and the Volterra operators of order $n \in \mathbb{N}^{*}$ defined in $\mathcal{V}_{n}$ by

$$
\mathrm{V}_{n}\left[u_{1}, \ldots, u_{n}\right](t):=\int_{\mathbb{T}^{n}} h_{n}\left(\tau_{1}, \ldots, \tau_{n}\right) \prod_{i=1}^{n} u_{i}\left(t-\tau_{i}\right) \mathrm{d} \tau_{i}
$$

where $h_{n}$ are called the Volterra kernels.

Note that equilibrium $y_{0}$ is usually chosen as zero, using the change of output $y \rightarrow y-y_{0}$. In the sequel, $y_{0}$ is kept non zero for sake of completeness, and all the $y_{n}$ 's are called nonlinear homogeneous order contributions (or orders for short).

It has been shown in Boyd and Chua (1985) that a Volterra series operator V can be used to approximate any fading-memory time-invariant continuous nonlinear system. In practice, bounds of convergence radius $\rho_{h}$ can be computed for large classes of well-posed systems (Brockett, 1977; Hélie \& Laroche, 2014).

Volterra kernels of a system are not unique: permuting time variables in $h_{n}$ preserves $y_{n}$ in (1). For identification issues, it is convenient to use uniquely-defined kernels (Rugh, 1981). Symmetric ones are those invariant with respect to permutations, i.e. such that for all permutations $\pi$ of $n$ variables,

$$
h_{n}\left(\tau_{\pi(1)}, \ldots, \tau_{\pi(n)}\right)=h_{n}\left(\tau_{1}, \ldots, \tau_{n}\right) .
$$

To improve identification of $\mathrm{V}$ from measurements, this paper exploits the following straightforward properties.

Property 1 (Operator $\mathrm{V}_{n}$ ). Let $\mathrm{V}_{n} \in \mathcal{V}_{n}$ as defined in (2). Then the following properties holds:

(i) Symmetry: if $h_{n}$ is symmetric (w.r.t. its time variables) then $\mathrm{V}_{n}$ is symmetric (w.r.t. its input signals); 
(ii) $n$-linearity: for all signals $u_{1}, \ldots, u_{n}, v$ and scalar $\alpha$,

$\mathrm{V}_{n}\left[u_{1}, \ldots, u_{k}+\alpha v, u_{k+1}, \ldots, u_{n}\right]=\mathrm{V}_{n}\left[u_{1}, \ldots, u_{n}\right]+\alpha \mathrm{V}_{n}\left[u_{1}, \ldots, v, u_{k+1}, \ldots, u_{n}\right]$,

with $1 \leq k \leq n$;

(iii) Homogeneity: for all signals $u_{1}, \ldots, u_{n}$ and scalar $\alpha$,

$$
\mathrm{V}_{n}\left[\alpha u_{1}, \ldots, \alpha u_{n}\right]=\alpha^{n} \mathrm{~V}_{n}\left[u_{1}, \ldots, u_{n}\right]
$$

In the sequel, kernels $h_{n}$ and operators $\mathrm{V}_{n}$ are considered symmetric and convergence conditions are assumed to be met.

\subsection{Amplitude-based Separation (AS) Method}

This section recalls the order separation method introduced in the 70's in Halme et al. (1971); Simpson and Power (1972).

Denote $\mathrm{V} \in \mathcal{V} \mathcal{S}$ the operator of a truncated Volterra series with degree $N$. Consider a reference input signal $u \in \mathcal{U}$ and a set of gains $\alpha_{k} \in \mathbb{R} \backslash\{0\}, k=0, \ldots, N$. Introduce the collection of input signals $u_{k}=\alpha_{k} u \in \mathcal{U}$ output signals $z_{k}=\mathrm{V}\left[u_{k}\right] \in \mathcal{Y}$. From (1) and (5), it follows that

$$
\begin{gathered}
{\left[\begin{array}{c}
z_{0} \\
z_{1} \\
\vdots \\
z_{N}
\end{array}\right](t)=\left[\begin{array}{cccc}
1 & \alpha_{0} & \ldots & \alpha_{0}^{N} \\
1 & \alpha_{1} & \ldots & \alpha_{1}^{N} \\
\vdots & \vdots & \ddots & \vdots \\
1 & \alpha_{N} & \ldots & \alpha_{N}^{N}
\end{array}\right] \cdot\left[\begin{array}{c}
y_{0} \\
y_{1} \\
\vdots \\
y_{N}
\end{array}\right](t)} \\
\boldsymbol{z}(t)= \\
\quad \boldsymbol{y}(t),
\end{gathered}
$$

where the $y_{n}$ 's are the homogeneous orders of the truncated series in response to the input signal $u$.

Since $\boldsymbol{A}$ is a Vandermonde matrix, it is invertible if and only if the $\alpha_{k}$ are all different. In this case, it is possible to recover orders $y_{n}$. In the following, this method will be referred as the Amplitude-based Separation (AS) method.

In practice, inverting the Vandermonde matrix $\boldsymbol{A}$ poses numerical difficulties due to its bad conditioning (Pan, 2016). Those can be partially alleviated by using Newton or Lagrange Recursive methods (Golub \& Van Loan, 2012, Algorithm 4.6.1 and 4.6.2), or considering more than $N+1$ gains (leading to more test signals) and using a pseudo-inverse matrix. Also, making use of several amplitudes and a unique waveform poses several limitations: for high amplitudes, the system can overload or be excited in a regime outside the Volterra series convergence domain; for small ones, higher orders are more and more hidden in measurement noise. Thus a trade-off has to be made between the condition number quality of $\boldsymbol{A}$ and the amplitude range narrowing. To the knowledge of the authors, the truncation order $N$ exceeds 5 in only one work (Zhang \& Billings, 2017).

To design a robust identification, the main idea of this paper is to exploit phase deconstruction/reconstruction in a preliminary order separation step. 


\section{Complex-valued Phase-based Separation (CPS)}

This section first presents ( $§ 3.1)$ some recalls on the phase exploitation for order separation in the case of 'abstract' (non physical) case of complex-valued inputs and outputs:

$$
\mathcal{U}_{\mathbb{C}}=\mathcal{Y}_{\mathbb{C}}:=L^{\infty}(\mathbb{T}, \mathbb{C}) .
$$

Second, in $\S 3.2$, the main properties of this method are analysed and stated.

\subsection{Method presentation}

Denote $\mathrm{V} \in \mathcal{V} \mathcal{S}$ the operator of a truncated Volterra series with degree $N$. Consider a reference input signal $u \in \mathcal{U}_{\mathbb{C}}$ and $w=e^{j 2 \pi /(N+1)}$ the first $(N+1)^{\text {th }}$ root of unity. Replacing gains $\alpha_{k}$ by $w^{k}, k=0, \ldots, N$ in the AS method leads to

$$
\begin{gathered}
{\left[\begin{array}{c}
z_{0} \\
z_{1} \\
\vdots \\
z_{N}
\end{array}\right](t)=\left[\begin{array}{cccc}
1 & 1 & \ldots & 1 \\
1 & w & \ldots & w^{N} \\
\vdots & \vdots & \ddots & \vdots \\
1 & w^{N} & \ldots & w^{N^{2}}
\end{array}\right]} \\
\boldsymbol{z}(t)= \\
\quad \boldsymbol{W}
\end{gathered}
$$

where $\boldsymbol{W}$ is the Discrete Fourier Transform (DFT) matrix of order $N+1$ with normalization factor 1. Its optimal conditioning makes the recovery of orders $\boldsymbol{y}(t)=\boldsymbol{W}^{-1} \boldsymbol{z}(t)$ robust, contrary to the AS method. Note that in (7), the DFT is applied to homogeneous orders $y_{n}$ (produced by the reference input signal $u=u_{0}$ ) with respect to order variable $n$ (and not the time variable). This method is referred below as the Complex-valued Phase-based Separation (CPS) method.

From the formal algebraic point view, AS and CPS methods look very similar. But from the signal point of view, they operate in very different ways: AS exploits amplitudes (whatever the signal shape) as a contrasting parameter of homogeneous orders on a validity range to be maximal, whereas CPS exploits shape (whatever the amplitude) through phase deconstruction and reconstruction. Differences on excitations can be summarized by comparing factors $\alpha_{k}$, $w^{k}$ and their power in the complex plane (see Fig. 2): in CPS, all the factors and their power lie on the unit circle and are one of the $N+1$ unit-roots; in AS, they all lie on the real axis and powers of $\alpha_{k}$ converge towards 0 (if $\left|\alpha_{k}\right|<1$ ) or diverge (if $\left|\alpha_{k}\right|>1$ ) as the truncation order $N$ increases.

\subsection{Properties of CPS method}

Due to the properties of the DFT, the CPS method comes with interesting properties.

\subsubsection{Zero-padding and number of phases}

As will be shown in $\S 3.2 .2$ and $\S 3.2 .3$, the number of phases $P$ has a positive impact on the robustness of the CPS method. For a given maximal order $N$, it can be artificially increased using zero-padding.

Let the number of phases $P$ be greater than $P_{\min }=N+1$. Introduce $w=e^{j 2 \pi / P}$, and the collection of input signals $u_{k}=w^{k} u \in \mathcal{U}_{\mathbb{C}}, k=0, \ldots, P-1$ and output signals $z_{k}=$ 


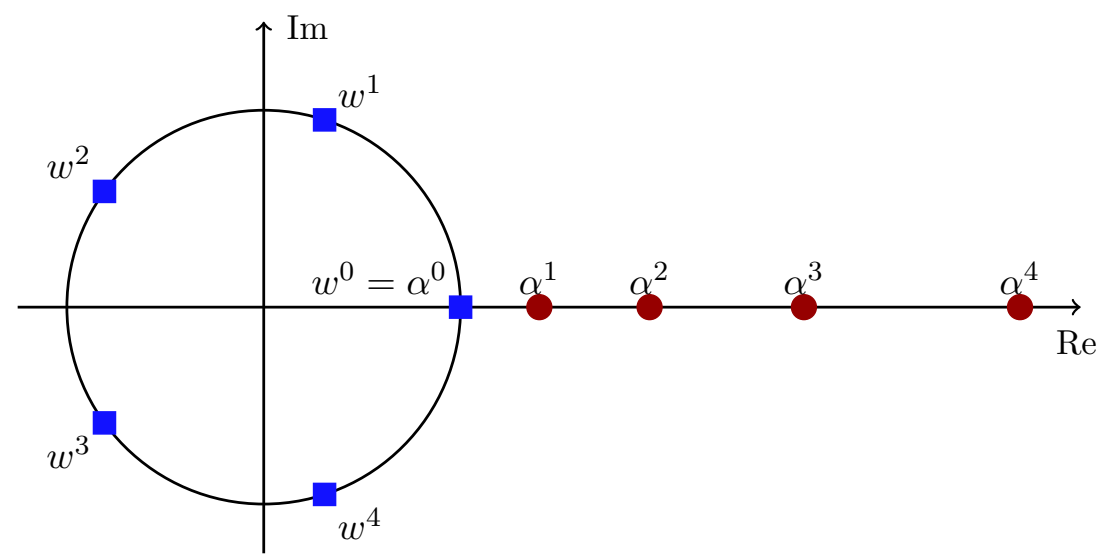

Figure 2. Powers of typical factors used in AS and CPS methods, compared in the complex plane: (AS) red circles (•) for $\alpha>1$; (CPS) blue squares (घ) for $w=e^{j 2 \pi /(N+1)}$ with $N=4$.

$\mathrm{V}\left[u_{k}\right] \in \mathcal{Y}_{\mathbb{C}}$. Then relation (7) remains valid, where $\boldsymbol{W}$ is now the DFT matrix of order $P$ and the vector of orders is $\boldsymbol{y}=\left[\begin{array}{llllll}y_{0} & \ldots & y_{N} & 0 & \ldots & 0\end{array}\right]^{T}$, with $P-N-1$ zeros. Considering more than $P_{\min }$ phases increases the number of tests signals $K$ (equal to $P$ for CPS method).

\subsubsection{Nonlinear order aliasing}

Now, consider that $\mathrm{V} \in \mathcal{V} \mathcal{S}$ is the operator of an infinite Volterra series. Consider a number of phases $P \geq P_{\min }=N+1$, where $N$ is the chosen approximation order. Since $w^{n+r P}=w^{n}$ for any $P^{\text {th }}$ root of unity $w$ and for all integers $n$ and $r$, property (5) yields

$$
\mathrm{V}[w u](t)=\sum_{n=0}^{P-1} w^{n} \sum_{r=0}^{\infty} y_{n+r P}(t),
$$

and (7) becomes

$$
\boldsymbol{z}(t)=\boldsymbol{W}\left(\boldsymbol{y}(t)+\sum_{r=1}^{\infty} \boldsymbol{y}^{(\boldsymbol{r})}(t)\right),
$$

with $\boldsymbol{y}=\left[\begin{array}{lll}y_{0} & \ldots & y_{P-1}\end{array}\right]^{T}$ and $\boldsymbol{y}^{(\boldsymbol{r})}=\left[\begin{array}{lll}y_{r P} & \ldots & y_{r P+P-1}\end{array}\right]^{T}$. This reveals that order $y_{n}$ is perturbed by a residual term $\sum_{r=1}^{\infty} y_{r P+n}$, which is structured as a nonlinear order aliasing. This effect permits to use the amplitude as a contrast factor to control the Signal-to-Residual Ratio (SRR).

Replacing gains $w^{k}$ by numbers $\gamma_{k}=\rho w^{k}$, with $0<\rho<1$, leads to

$$
\boldsymbol{z}(t)=\boldsymbol{W} \cdot \boldsymbol{R} \cdot\left(\boldsymbol{y}(t)+\sum_{r=1}^{\infty} \rho^{r P} \boldsymbol{y}^{(\boldsymbol{r})}(t)\right),
$$

with $\boldsymbol{R}:=\operatorname{diag}\left(\rho^{0}, \rho^{1}, \ldots, \rho^{P-1}\right)$.

Therefore, in estimated orders $\widetilde{\boldsymbol{y}}(t)=\boldsymbol{R}^{-1} \boldsymbol{W}^{-1} \boldsymbol{z}(t)$, there is a ratio $1 / \rho^{P}$ between the desired signal and the first residual $\boldsymbol{y}^{(1)}(t)$. This ratio permits to improve the SRR by choosing a high $P$ or a low contrast factor $\rho$. For example, a SRR gain of $20 \mathrm{~dB}$ is obtained by setting $P=100$ and $\rho \approx 0.977$. 


\subsubsection{Noise reduction}

Suppose that each measured output $z_{k}$ is perturbed by an independent and identically distributed (i.i.d.) complex Gaussian noise, i.e.

$$
\widehat{\boldsymbol{z}}(t)=\boldsymbol{z}(t)+\boldsymbol{\varepsilon}_{\boldsymbol{r}}(t)+j \boldsymbol{\varepsilon}_{\boldsymbol{i}}(t)
$$

where $\varepsilon_{r}$ and $\varepsilon_{i}$ are both multivariate i.i.d. Gaussian noise of null mean and covariance matrices $\boldsymbol{\Sigma}_{\boldsymbol{r} / \boldsymbol{i}}=\sigma_{r / i}^{2} \boldsymbol{I}$, with $\mathcal{I}$ the identity matrix. The best estimator $\widehat{\boldsymbol{y}}$ of $\boldsymbol{y}$ in a least meansquares sense is

$$
\widehat{\boldsymbol{y}}(t)=\boldsymbol{R}^{-1} \boldsymbol{W}^{-1} \widehat{\boldsymbol{z}}(t)=\widetilde{\boldsymbol{y}}(t)+\widehat{\boldsymbol{\varepsilon}}_{\boldsymbol{r}}(t)+j \widehat{\boldsymbol{\varepsilon}}_{\boldsymbol{i}}(t),
$$

where $\widehat{\varepsilon}_{r / i}$ are multivariate i.i.d. Gaussian noise of null mean and covariance matrices

$$
\widehat{\boldsymbol{\Sigma}}_{r / i}=\frac{\sigma_{r / i}^{2}}{P} \boldsymbol{R}^{-2}
$$

Equation (13) shows that using CPS method, Signal-to-Noise Ratio (SNR) between estimated order $y_{n}$ and error due to noise measurement is reduced by a factor $\rho^{n} \sqrt{P}$. For example, setting $P$ and $\rho$ as in the previous example (§ 3.2.2) and considering a maximum order of $N=9$ leads to a SNR gain going from $20 \mathrm{~dB}$ (for the zeroth order) to $18.2 \mathrm{~dB}$ (for order 9 ). This illustrates how increasing the number of phases $P$ (and therefore the number of test signals $K$ ) only leads to a more robust order separation method.

But the CPS method is abstract and useless in practice since only real-valued signals can be used on a physical system. Its recast from complex-valued to real-valued signals is not possible because equation (7) cannot be recomposed using an independent processing of real and imaginary parts.

\section{Real-valued Phase-based Separation (RPS)}

This section provides a method based on the phase exploitation, adapted to the use of realvalued signals for handling real-life cases:

$$
\mathcal{U}=\mathcal{Y}:=L^{\infty}(\mathbb{T}, \mathbb{R})
$$

The main theoretical result of this paper is stated in Theorem 4.4 ( $\$ 4.1)$. It allows the derivation of the main practical result (RPS method, $\S 4.2$ ), the properties of which are detailed in $\S 4.3$.

\subsection{Preliminary results}

In this section, let $u \in \mathcal{U}_{\mathbb{C}}$ be a complex-valued signal, and operators $\mathrm{V}_{n} \in \mathcal{V}_{n}$ be associated with symmetric real-valued Volterra kernels $h_{n}$.

Definition 4.1 (Interconjugate signal). The interconjugate signals $y_{n, q} \in \mathcal{Y}_{\mathbb{C}}, q=0, \ldots, n$, 
are defined by

$$
y_{n, q}(t):=\mathrm{V}_{n}[\underbrace{u, \ldots, u}_{n-q \text { times }}, \underbrace{\bar{u}, \ldots, \bar{u}}_{q \text { times }}](t),
$$

where $\bar{a}$ denotes the complex conjugate of $a$. By extension, we also denote $y_{0,0}(t):=y_{0}$.

Remark 1. Signals $y_{n, 0}$ (respectively $y_{n, n}$ ) correspond to the output of order $n$ of a series excited by signal $u$ (resp. $\bar{u}$ ). Signals $y_{n, q}$ with $0 \neq q \neq n$ do not have similar correspondence in terms of input/output relationships.

Property 2. By symmetry of $\mathrm{V}_{n}$, it is straightforward to show that $y_{n, n-q}(t)=\overline{y_{n, q}}(t)$, and that for even $n$, signal $y_{n, n / 2}$ is real.

Interconjugate signals allows the rewriting of the output of order $n$ for a real-valued input signal, as the following lemma shows.

Lemma 4.2. Let $r=\operatorname{Re}[u] \in \mathcal{U}$. Then $y_{n}=\mathrm{V}_{n}[r, \ldots, r] \in \mathcal{Y}$ is given by

$$
y_{n}(t)=\frac{1}{2^{n}} \sum_{q=0}^{n}\left(\begin{array}{l}
n \\
q
\end{array}\right) y_{n, q}(t),
$$

where $\left(\begin{array}{l}n \\ q\end{array}\right)=\frac{n !}{q !(n-q) !}$ is the binomial coefficient.

Proof. The proof stems from the symmetry and $n$-linearity of operators $\mathrm{V}_{n}$ fed by $r(t)=$ $(u(t)+\bar{u}(t)) / 2$.

Example 4.3. Consider the cosinusoidal signal $r(t)=\cos (2 \pi f t)$ and its analytic version $u(t)=e^{j 2 \pi f t}$. Interconjugate signals $y_{n, q}$ are complex sinusoids of frequency $(n-2 q) f$, with phase and amplitude depending on operator $\mathrm{V}_{n}$. Furthermore, if $\mathrm{V}_{n}$ is such that $\mathrm{V}_{n}[r, \ldots, r](t)=r(t)^{n},(15)$ leads back to the Chebyshev expansion of $\cos (2 \pi f t)^{n}$.

Lemma 4.2 shows that separating orders $y_{n}$ could be achieved from the knowledge of all terms $y_{n, q}$. To this end, a first solution has been proposed in Bouvier et al. (2017). It combines phase deviations (as in CPS) and amplitude gains (as in AS). In Bouvier et al. (2018), a notion of homophase signals is introduced and used to derive an iterative identification method. To reject numerical difficulties due to Vandermonde matrices and reserve the amplitude of inputs as a free parameter, a better alternative for order separation is proposed below. It involves linear combinations of pairs of signals with various phase deviations, according to the following theorem.

Theorem 4.4. Consider a Volterra series with a maximal order $N \in \mathbb{N} \cup\{+\infty\}$. Denote $\theta_{i}, i=1,2$ two complex scalars on the unit circle, and let $y \in \mathcal{Y}$ be the output of the series excited by $2 \operatorname{Re}\left[\left(\theta_{1}+\theta_{2}\right) u\right] \in \mathcal{U}$. Then

$$
y(t)=\sum_{\left|p_{1}\right|+\left|p_{2}\right| \leq N} \theta_{1}^{p_{1}} \theta_{2}^{p_{2}} \psi_{p_{1}, p_{2}}^{(N)}(t),
$$


with, $\forall\left(p_{1}, p_{2}\right) \in \mathbb{Z}^{2}$,

$$
\psi_{p_{1}, p_{2}}(t):=\sum_{\substack{n=\left|p_{1}\right|+\left|p_{2}\right| \\ n \equiv\left(p_{1}+p_{2}\right)[2]}}^{N} \kappa_{\left|p_{1}\right|,\left|p_{2}\right|}^{(n)} y_{n, \frac{n-p_{1}-p_{2}}{2}}(t),
$$

where where $a \equiv b[k]$ stands for ' $a$ and $b$ are congruent modulo $k$ ' and where

$$
\kappa_{a_{1}, a_{2}}^{(n)}:=\sum_{\substack{l=a_{1} \\
l \equiv a_{1}[2]}}^{n-a_{2}}\left(\begin{array}{c}
n \\
\frac{l+a_{1}}{2}, \frac{n-l+a_{2}}{2}, \frac{l-a_{1}}{2}, \frac{n-l-a_{2}}{2}
\end{array}\right),
$$

with $\left(\begin{array}{c}n \\ q_{1}, q_{2}, q_{3}, q_{4}\end{array}\right)=\frac{n !}{q_{1} ! q_{2} ! q_{3} ! q_{4} !}$ the multinomial coefficient.

Proof. See Appendix A.

Corollary 4.5 (Symmetries on $\left.\psi_{p_{1}, p_{2}}\right)$. Using Property 2 and $\kappa_{a_{1}, a_{2}}^{(n)}=\kappa_{a_{2}, a_{1}}^{(n)}$, the following properties holds:

(i) equality: $\psi_{p_{1}, p_{2}}=\psi_{p_{2}, p_{1}}$

(ii) conjugation: $\psi_{-p_{1},-p_{2}}=\overline{\psi_{p_{1}, p_{2}}}$ and $\psi_{p,-p}$ is real.

Theorem 4.4 shows how the output of a Volterra series can be decomposed onto a 2dimensional phase plane $\left(p_{1}, p_{2}\right)$ (see Fig. 3). This makes possible to separate signals $\psi_{p_{1}, p_{2}}$ using a 2-dimensional DFT. Furthermore, this repartition exhibits the tetrahedron structure of a Pascal's pyramid, which is due to the multilinearity of the $\mathrm{V}_{n}$ operators (see Fig. 4). This property allows the extraction of signals $y_{n, q}$ from $\psi_{p_{1}, p_{2}}$. Those two steps constitute the main part of the proposed separation method, which is now detailed.

\subsection{RPS Method}

Denote $\mathrm{V} \in \mathcal{V} \mathcal{S}$ the operator of a truncated Volterra series with degree $N$, and $u \in \mathcal{U}_{\mathbb{C}}$ a complex-valued reference signal. Let $u_{R}=\operatorname{Re}[u] \in \mathcal{U}$ the associated real-valued excitation signal, and $y_{n}=\mathrm{V}_{n}\left[u_{R}\right] \in \mathcal{Y}$ the homogeneous orders we want to separate.

(i) Creation of the signals collection: Let $P=2 N+1$ and $w=e^{j 2 \pi / P}$. Introduce the collection of input signals $U_{k_{1}, k_{2}}=2 \operatorname{Re}\left[\left(w^{k_{1}}+w^{k_{2}}\right) u\right] \in \mathcal{U}, k_{1}, k_{2}=0, \ldots, P-1$, and output signals $Z_{k_{1}, k_{2}}=\mathrm{V}\left[U_{k_{1}, k_{2}}\right] \in \mathcal{Y}$.

(ii) Separation of signals $\psi_{p_{1}, p_{2}}$ : Introduce the matrix $\Psi$ such that $\psi_{p_{1}, p_{2}}(t)=$ $\boldsymbol{\Psi}_{p_{1}[P], p_{2}[P]}(t), \forall p_{1}, p_{2}=-N, \ldots, N$. Let $\mathcal{F}_{\mathrm{P}, \mathrm{P}}$ denote the 2D-DFT operator ${ }^{3}$ of or$\operatorname{der}(P, P)$. Then Theorem 4.4 gives

$$
\boldsymbol{Z}(t)=\mathcal{F}_{\mathrm{P}, \mathrm{P}}\{\boldsymbol{\Psi}\}(t)
$$

\footnotetext{
${ }^{3}$ As for the one dimensional DFT, the normalization factor is put in the inverse operator, meaning that

$$
\mathcal{F}_{\mathrm{M}, \mathrm{N}}\{\boldsymbol{x}\}_{k, l}=\sum_{m=0}^{M-1} \sum_{n=0}^{N-1} x_{m, n} e^{-j 2 \pi\left(\frac{k m}{M}+\frac{l n}{N}\right)},
$$
}

where $\boldsymbol{x}$ is a two-dimensional signal in matrix form. 


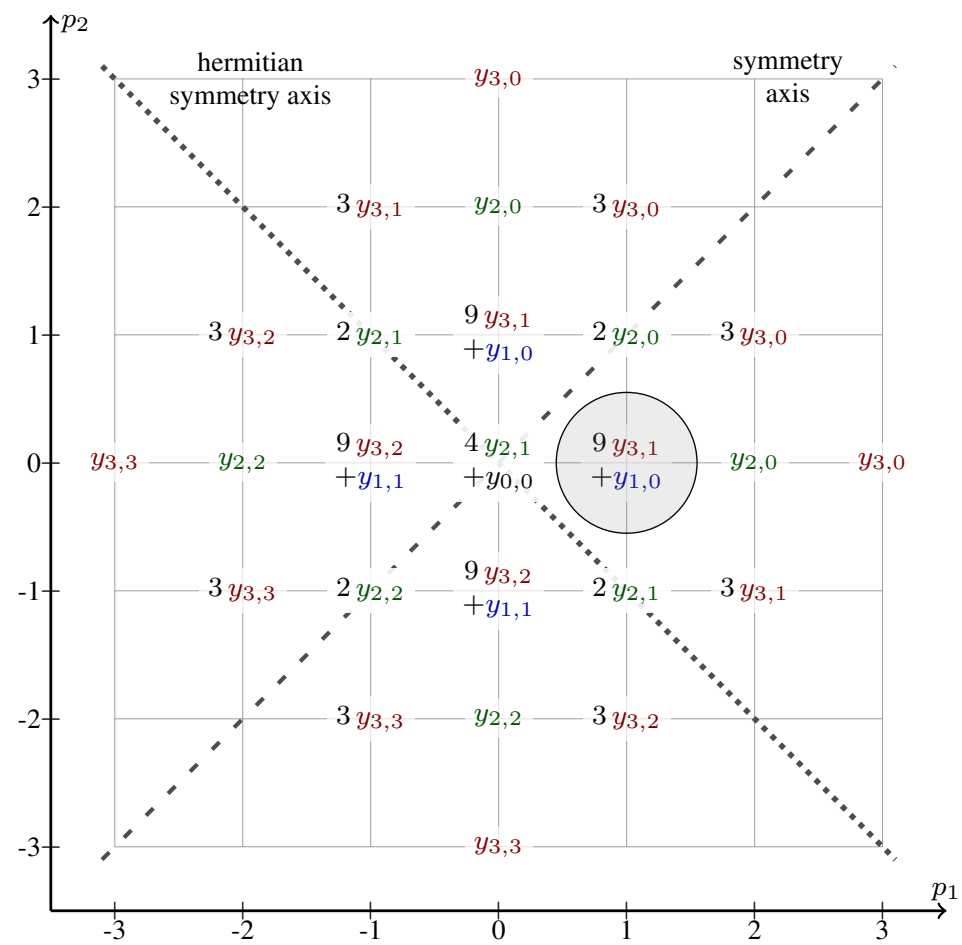

Figure 3. Repartition of interconjugate signals $y_{n, q}$ up to order 3 in the $2 \mathrm{D}$ phase plane $\left(p_{1}, p_{2}\right)$; the dashed line (- -) corresponds to the symmetry axis, and the dotted line ( $\ldots)$ to the hermitian symmetry axis; e.g. the gray circle corresponds to the location of $\psi_{1,0}$

Signals $\psi_{p_{1}, p_{2}}$ are thus recovered using an inverse 2D-DFT.

(iii) Separation of interconjugate signals $y_{n, q}$ : Let

$\phi_{p}$ $\left[\begin{array}{llll}\psi_{r_{0}, r_{Q}} & \psi_{r_{1}, r_{Q-1}} & \cdots & \psi_{r_{Q}, r_{0}}\end{array}\right]^{T}$ regroup all signals $\psi_{p_{1}, p_{2}}$ on the anti-diagonal $p_{1}+p_{2}=p$, where $Q=N$ if $p \equiv N[2], Q=N-1$ otherwise, and $r_{j}=\left\lfloor\frac{p+N}{2}\right\rfloor-j$, with $\lfloor$. denoting the integer part. Also let $\boldsymbol{v}_{\boldsymbol{p}}=\left[\begin{array}{llll}y_{Q, \frac{Q-p}{2}} & y_{Q-2, \frac{Q-p}{2}-1} & \ldots & y_{|p|, \frac{|p|-p}{2}}\end{array}\right]^{T}$ regroup all interconjugate signals $y_{n, q}$ with $q=\frac{n-p}{2}$. Then, using (17), the following relationship holds:

$$
\phi_{\boldsymbol{p}}(t)=\boldsymbol{\Lambda}_{\boldsymbol{p}} \boldsymbol{v}_{\boldsymbol{p}}(t)
$$

where

$$
\boldsymbol{\Lambda}_{p}=\left[\begin{array}{cccc}
\kappa_{r_{0}, r_{Q}}^{(Q)} & 0 & \ldots & 0 \\
\kappa_{r_{1}, r_{Q-1}}^{(Q)} & \kappa_{r_{1}, r_{Q-1}}^{(Q-2)} & \ldots & 0 \\
\vdots & \vdots & \ddots & \vdots \\
\kappa_{r_{Q / 2}, r_{Q / 2}}^{(Q)} & \kappa_{r_{Q / 2}, r_{Q / 2}}^{(Q-2)} & \ldots & \kappa_{r_{Q / 2}, r_{Q / 2}}^{(|p|)} \\
\vdots & \vdots & . & \vdots \\
\kappa_{r_{Q}, r_{0}}^{(Q)} & 0 & \ldots & 0
\end{array}\right]
$$




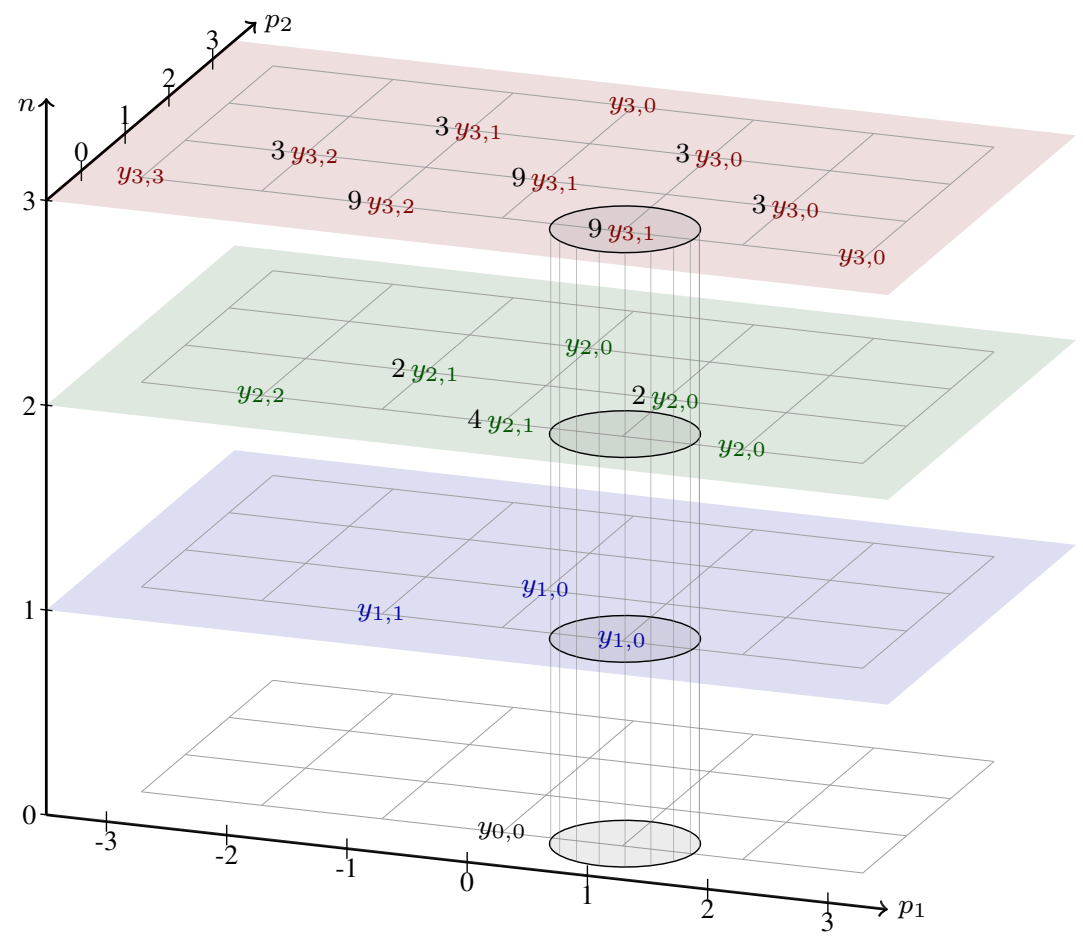

Figure 4. Repartition of interconjugate signals $y_{n, q}$ up to order 3 in the $3 \mathrm{D}$ half-space $\left(p_{1}, p_{2}, n\right)$, with $p_{2} \geq 0$; the gray cylinder of axis $\left(p_{1}=1, p_{2}=0\right)$ regroups all terms constitutive of $\psi_{1,0}$.

for $p$ even, and

$$
\boldsymbol{\Lambda}_{\boldsymbol{p}}=\left[\begin{array}{ccc}
\kappa_{r_{0}, r_{Q}}^{(Q)} & \ldots & 0 \\
\vdots & \ddots & \vdots \\
\kappa_{r_{(Q-1) / 2}, r_{(Q+1) / 2}}^{(Q)} & \ldots & \kappa_{r_{(Q)-1) / 2}, r_{(Q+1) / 2}}^{(|p|)} \\
\kappa_{r_{(Q+1) / 2}, r_{(Q-1) / 2}}^{(Q)} & \ldots & \kappa_{r_{(Q+1) / 2}, r_{(Q-1) / 2}}^{(|p|)} \\
\vdots & . & \vdots \\
\kappa_{r_{Q}, r_{0}}^{(Q)} & \ldots & 0
\end{array}\right]
$$

for $p$ odd. Inverting (20) for all $p=-N, \ldots, N$ gives all signals $y_{n, q}, n=0, \ldots, N, q=$ $0, \ldots, n$.

(iv) Reconstruction of orders $y_{n}$ : Once the interconjugate signals $y_{n, q}$ are known, homogeneous orders $y_{n}$ can be reconstructed using (15).

Those four steps constitute the proposed method, which will be referred as the Real-valued Phase-based Separation (RPS) method. In practice, one does not need to make $P^{2}$ measurements, due to the symmetry $U_{k_{1}, k_{2}}=U_{k_{2}, k_{1}}$. So the number of test signals is only $K=\frac{P(P+1)}{2}=(2 N+1)(N+1)$ (number of terms in the triangular part of matrix $\boldsymbol{Z}$ ), and the remaining signals of matrix $\boldsymbol{Z}$ are found by symmetry.

Robustness of RPS method greatly depends on the numerical conditioning of matrices $\Lambda_{p}$. This value is fixed for a given truncation order $N$ and phase index $p$, and increases with $N$ and $|p|$. Therefore, as for AS method, RPS robustness will decrease when $N$ is large. 


\subsection{Properties of the RPS method}

\subsubsection{Zero-padding and number of signals}

As in CPS method, it is possible to use a number of phases $P$ greater than the required minimum $P_{\min }=2 N+1$. As long as $P \geq P_{\min }$ and the truncation hypothesis is valid, retrieving signals $\psi_{p_{1}, p_{2}}$ from $\boldsymbol{\Psi}$ is possible.

\subsubsection{Nonlinear order aliasing}

Now consider that $\mathrm{V} \in \mathcal{V} \mathcal{S}$ is the operator of an infinite Volterra series. As in CPS, a nonlinear order aliasing appears in step (ii). Here it is manifested by the fact that the desired signal $\psi_{p_{1}, p_{2}}$ is mingled with all $\psi_{i, j}$ such that $p_{1} \equiv i[P]$ and $p_{2} \equiv j[P]$, deteriorating its estimation. The only way to minimize this aliasing is to choose $P \gg 2 N+1$.

It would also be interesting to diminish the effect of higher-order residuals $y_{n, q}, n>N$ in signals $\psi_{p_{1}, p_{2}}$. Unfortunately, the use of a contrast factor as described in $\S 3.2 .2$ is not feasible for RPS. Indeed, because $\psi_{p_{1}, p_{2}}$ contains contributions from all orders $n \geq\left|p_{1}\right|+\left|p_{2}\right|$ such that $p_{1}+p_{2} \equiv n[2]$, the SRR gain would be $1 / \rho^{2}$.

\subsubsection{Number of phases and noise reduction}

Suppose that each measured output $Z_{k_{1}, k_{2}}$ is perturbed by an i.i.d. complex Gaussian noise. Identical developments as in $\S 3.2 .3$ show that the use of a 2D-DFT improves the SNR in estimation of signals $\psi_{p_{1}, p_{2}}$ by a factor $P$. But the independence hypothesis between noise is false, since $Z_{k_{1}, k_{2}}$ and $Z_{k_{2}, k_{1}}$ comes from the same measurement (with the same noise). Similar developments as in $\S 3.2 .3$ shows that the SNR improvement has the same order of magnitude, but the error noise between signals $\psi_{p_{1}, p_{2}}$ is not independent (i.e. the noise correlation matrix is not diagonal). This shows that, for a given maximal order $N$, a large value of $P$ allows a better estimation of signals $\psi_{p_{1}, p_{2}}$ in step (ii), and therefore of $y_{n, q}$ and $y_{n}$ in steps (iii-iv). However a large $P$ leads to a large number of test signals $K$.

\subsubsection{Amplitude of the input collection}

All signals in the input collection $U_{k_{1}, k_{2}}$ do not have the same amplitude. It greatly depends on the chosen signal $u$, and also, for a given $u$, on the factor $a=\left|2\left(w^{k_{1}}+w^{k_{2}}\right)\right|$.

The maximum $a_{\max }$ is reached for $k_{1}=k_{2}$ and is equal to 4 . The minimum $a_{\min }$ is reached when the two values $w^{k_{1}}$ and $w^{k_{2}}$ are the furthest apart on the complex circle, i.e $\left|k_{1}-k_{2}\right|=$ $(P-1) / 2$ for an odd ${ }^{4} P$. Basic calculus shows that $a_{\min }=4 \cos \left(\frac{\pi}{2} \frac{P-1}{P}\right)$.

Therefore the ratio between maximum and minimum amplitudes in the input collection will be closely related to $\cos \left(\frac{\pi}{2} \frac{P-1}{P}\right)^{-1}$. Therefore, increasing the number of phases $P$ also increases the amplitude range needed for the excitation of the system ${ }^{5}$. For example, setting $P=19$ (i.e. minimum number of phases when considering a maximum order $N=9$ ) leads to an amplitude range of $\sim 21.7 \mathrm{~dB}$.

\footnotetext{
${ }^{4}$ An even $P$ leads to $a_{\min }=0$ and must be avoided.

${ }^{5}$ It is important to note that using RPS, the amplitude variations between all signals of the input collection are not wanted and chosen (as in AS method) but are a side effect of the method.
} 


\subsubsection{Computational complexity}

Because RPS method relies only on linear algebra operations, it is easy to compute its computational complexity ${ }^{6}$. In the following, we consider the case of a (discrete-time) signal of length $L \gg P \geq 2 N+1$ (i.e. the number of data points in the signal is much greater than the number of phases used, which is a reasonable assumption).

Step (ii) consists of the application of an inverse 2D-DFT at each time step. Using an implementation relying on one-dimensional Fast-Fourier Transforms, the 2D-DFT has a complexity of $\mathcal{O}\left(P^{2} \log P^{2}\right)$. Therefore the complexity of step (ii) is $\mathcal{O}\left(2 L P^{2} \log P\right)$.

Step (iii) consists in solving (20) at each time step for all phases $p=-N, \ldots, N$, i.e. $2 N+1$ times. Matrices $\Lambda_{p}$ have different sizes, but $\Lambda_{0}$ is always the largest with size $(N+1) \times$ $(\lfloor N / 2\rfloor+1)$. Because $L \gg N$, the pseudo-inversion of $\boldsymbol{\Lambda}_{p}$ is negligible in terms of complexity compared to the products $\Lambda_{p}^{\dagger} \phi_{p}(t)$ for all time steps, where $\Lambda_{p}^{\dagger}$ denotes the pseudo-inverse of $\boldsymbol{\Lambda}_{\boldsymbol{p}}$. Therefore the complexity of step (iii) is $\mathcal{O}((2 N+1)(N+1)(\lfloor N / 2\rfloor+1) L)=$ $\mathcal{O}\left(L N^{3}\right)$. The reconstruction of orders in step (iv) can be shown to have a complexity of $\mathcal{O}\left(L N^{2}\right)$, which is negligible compared to step (iii).

The total complexity of RPS method is therefore $\mathcal{O}\left(\max \left(2 L P^{2} \log P, L N^{3}\right)\right)$. If $\log P>$ $N$, this complexity is dominated by the first term and hence determined by the number of phases $P$; otherwise it is cubic in the truncation order $N$. In both cases, complexity is linear with respect to the signal's length. In comparison, AS method has a complexity of $\mathcal{O}\left(L N^{2}\right)$. In practice, because $N$ and $P$ are not extremely large, computation times for RPS are very short, and close to those of AS.

\section{Least-squares identification based on separation}

This section presents, in the Least-Square (LS) formalism, how the homogeneous orders $y_{n}$ or the interconjugate signals $y_{n, q}$ can be used instead of the direct output $y$ for discrete-time Volterra kernel estimation. The ideas behind processes (A), (B) and (C) presented in $\S 5.2$ could easily be conveyed to other identification methods.

Discrete-time signals and systems are now considered. All previous definitions and results are available by replacing $\mathbb{T}$ by $\mathbb{T}_{d}:=\mathbb{Z}, \mathbb{N}$ or a subset $[0, L]_{\mathbb{N}}$ with $L \in \mathbb{N}^{*}$, and replacing integrals in (2) by discrete sums.

\subsection{Least-squares formulation and combinatorial matrix}

Suppose that the system-to-be-identified has a finite memory of $M$ samples. Given $L$ input-outputs measurements of the system to identify, relations (1) and (2) can be summarized in vectorial form by $\boldsymbol{y}=\boldsymbol{C f}$, where $\boldsymbol{y}$ is the column vector of output data, $f=\left[\begin{array}{lll}f_{0} & \ldots & f_{N}\end{array}\right]^{T}$ a column vector regrouping all kernel coefficients, and $\boldsymbol{C}=$ $\left[\begin{array}{lll}C_{0} & \ldots & C_{N}\end{array}\right]$ the input combinatorial matrix containing all the delayed-products of the input signal (see Rugh (1981) for more details about construction of this matrix). In order to reduce the number of coefficients to estimate, only non-redundant terms of the symmetric form $^{7}$ of kernels $h_{n}$ are considered in vectors $\boldsymbol{f}_{\boldsymbol{n}}$.

\footnotetext{
${ }^{6}$ The memory usage of RPS method will not be addressed here; it is generally not an issue, and computation can be done separately for each time step.

${ }^{7}$ Or equivalently the nonzero terms of the triangular form.
} 
Example 5.1. For an order 2 kernel of memory length $M=3, \boldsymbol{C}_{\mathbf{2}}$ and $\boldsymbol{f}_{\mathbf{2}}$ are given by ${ }^{8}$

$$
\boldsymbol{C}_{\mathbf{2}}=\left[\begin{array}{cccc}
u[0]^{2} & u[1]^{2} & u[2]^{2} & \ldots \\
0 & u[1] u[0] & u[2] u[1] & \ldots \\
0 & 0 & u[2] u[0] & \ldots \\
0 & u[0]^{2} & u[1]^{2} & \ldots \\
0 & 0 & u[1] u[0] & \ldots \\
0 & 0 & u[0]^{2} & \ldots
\end{array}\right]^{T} \text { and } \boldsymbol{f}_{\mathbf{2}}=\left[\begin{array}{c}
h_{2}[0,0] \\
h_{2}[0,1] \\
h_{2}[0,2] \\
h_{2}[1,1] \\
h_{2}[1,2] \\
h_{2}[2,2]
\end{array}\right]
$$

Similarly, a vectorial form of (14) is given by $y_{n, q}=C_{n, q} f_{n}$, where $C_{n, q}$ is the input combinatorial matrix corresponding to the column vector of interconjugate signal $\boldsymbol{y}_{n, q}$. Matrix $C_{n, q}$ is constructed in a similar way as $C_{n}$, but conjugated terms have to be taken into account when doing symmetrization ${ }^{9}$.

Example 5.2. For an order 2 kernel of memory length $M=3$, combinatorial matrix $\boldsymbol{C}_{\mathbf{2}, \mathbf{1}}$ is given by

$$
\boldsymbol{C}_{\mathbf{2 , 1}}=\left[\begin{array}{cccc}
|u[0]|^{2} & \frac{|u[1]|^{2}}{u[1] \overline{u[0]}+\overline{u[1]} u[0]} & \frac{\left.u[2]\right|^{2}}{2} & \ldots \\
0 & \frac{u[1]+\overline{u[2]} u[1]}{2} & \ldots \\
0 & 0 & \frac{u[2] \overline{u[0]}+\overline{u[0]} u[2]}{2} & \ldots \\
0 & |u[0]|^{2} & \frac{|u[1]|^{2}}{2[1] \overline{u[0]}+\overline{u[1]} u[0]} & \ldots \\
0 & 0 & \left.\ldots u[0]\right|^{2} & \ldots
\end{array}\right]^{T}
$$

\subsection{Identification methods}

(A) Direct estimation: Identifying kernels directly from the measured output comes down to solving $\boldsymbol{y}=\boldsymbol{C} \boldsymbol{f}$. This requires that the data length $L$ is larger than the number of coefficients to estimate, i.e. $L \geq\left(\begin{array}{c}M+N \\ N\end{array}\right)$ (see (Mathews \& Sicuranza, 2000)).

(B) Estimation on homogeneous orders: If the homogeneous orders $y_{n}$ are available, kernel estimation is done by solving $\boldsymbol{y}_{n}=\boldsymbol{C}_{n} \boldsymbol{f}_{n}$ for each order $n$. Signal length must fulfill the condition $L \geq\left(\begin{array}{c}M+n-1 \\ n\end{array}\right)$.

(C) Estimation on interconjugate signals: If the interconjugate signals $y_{n, q}$ are available, kernel estimation is done by solving, for each order $n$,

$$
\left[\begin{array}{c}
y_{n, 0} \\
\vdots \\
y_{n, n}
\end{array}\right]=\left[\begin{array}{c}
C_{n, 0} \\
\vdots \\
C_{n, n}
\end{array}\right] f_{n}
$$

Signal length must fulfill the condition $L \geq \frac{1}{n+1}\left(\begin{array}{c}M+n-1 \\ n\end{array}\right)$.

In those methods, computation time and memory usage directly depends on the size of the matrix to be inverted (and also varies with the algorithm used for solving the linear problem).

\footnotetext{
${ }^{8}$ The lexicographical order is used here, but other choices can be made, without impact on the identification.

${ }^{9}$ For example, combinatorial terms $u[l] \overline{u[l-1]}$ and $u[l-1] \overline{u[l]}$ are both linked to the same kernel coefficient $h_{2}[0,1]$, and should thus contribute equally to the corresponding term in $\boldsymbol{C}_{\mathbf{2}, \mathbf{1}}$.
} 
Size of matrix $C$ grows linearly with the data length $L$ but exponentially with the truncation order $N$ and system memory $M$, which makes this method quite cumbersome (time and memory-wise) when high nonlinearities and/or long memory are required.

Matrices $C_{n}$ have the same number of rows as $C$, but the number of columns, which grows with the current order $n$ as well as memory $M$, is always smaller. Therefore, the inversion of $C_{n}$ has a computation time and memory usage reduced compared to the inversion of $C$. Furthermore, we have observed in practice that method (B) in total (i.e. inversions of all $C_{n}$ done sequentially) is computationally quite faster than (A).

Each matrix $\boldsymbol{C}_{n, q}$ has the same size as $\boldsymbol{C}_{\boldsymbol{n}}$. But, for method (C), the matrix to invert for each order has a number of rows $n+1$ times higher. Therefore the computation time and memory usage of method $(\mathrm{C})$ is higher than method (B). Compared to method (A), the augmentation in the number of rows is accompanied by a reduction in the number of columns. In terms of computation time, the latter compensates the former when $M$ is large enough (for a given $N$ ). Furthermore, we have observed in practice that method (C) (i.e. resolution of (23) done sequentially for each order $n$ ) is computationally quite faster than (A).

\section{Evaluation and comparison}

This section evaluates the proposed methods using numerical simulation experiments. The code used for the separation and identification methods is available in an open-source Python toolbox ${ }^{10}$.

\subsection{Order separation evaluation}

Experiment description The simulated system is a discrete-time Volterra series truncated to order 9 , with a finite memory length of $M=5$ samples. Kernels values are set according to the following formula:

$$
h_{n}\left[k_{1}, \ldots, k_{n}\right]=10^{1-n}(-1)^{\sum_{i} k_{i}} e^{-\frac{\max k}{10}}
$$

Those choices are arbitrarily made in order to have (a) exponentially decaying kernels, (b) amplitude of orders $y_{n}$ decreasing w.r.t. $n$ and (c) a relatively low number of parameters to estimate (i.e. 2001). Real-life systems can be expected to meet criteria (a) and (b), but memory length is usually longer.

AS and RPS methods are compared using the standard relative Root-Mean-Square (RMS) error value between true and estimated order, given for each order $n$ by $\epsilon_{n}=$ $\operatorname{RMS}\left(\tilde{y}_{n}-y_{n}\right) / \operatorname{RMS}\left(y_{n}\right)$, where $\tilde{y}_{n}$ is the estimation of order $y_{n}$.

For RPS method, a complex white Gaussian noise of zero-mean and unit variance is used as the reference input signal $u$, with a length of $L=5000$ samples. For AS, the signal $4 \operatorname{Re}[u]$ is used as the input reference ${ }^{11}$. A perturbation noise (also white and Gaussian) is added to the measured outputs, with a SNR of $60 \mathrm{~dB}$ w.r.t. to the maximum measured RMS level. For each method, 10 experiments with different realization of the signal $u$ are done; presented results are the median over all experiments.

In order to have a meaningful comparison between AS and RPS, the same number of test signals $K=190$ is used for both methods. This corresponds to the required minimum

\footnotetext{
${ }^{10}$ https://github.com/d-bouvier/pyvi

${ }^{11}$ The factor 4 is here so that both collection of test signals have the same maximum amplitude.
} 


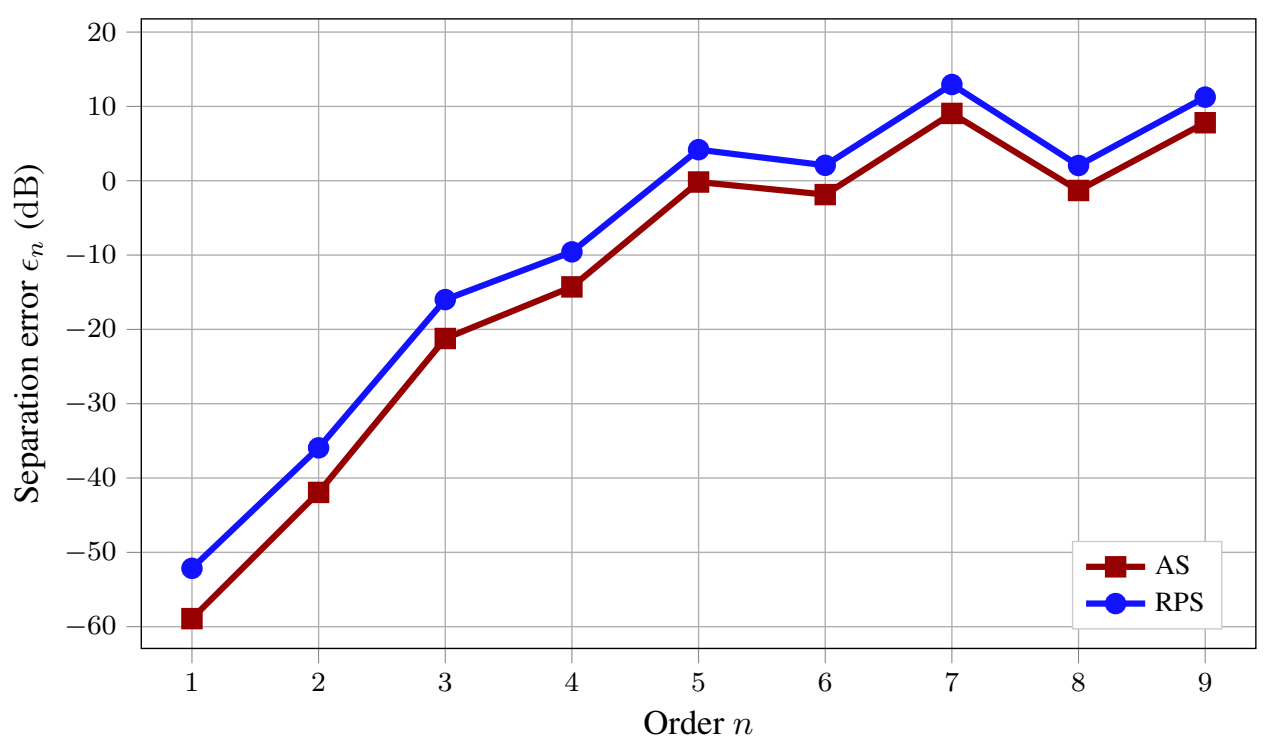

Figure 5. Comparison of separation error w.r.t order $n$ for methods AS ( $\bullet-)$ and RPS ( $\bullet-)$. Median values over all experiments are represented.

number by the RPS method. Furthermore, for AS method, gains $\alpha_{k}$ are chosen such that $\alpha_{k}=(-1)^{k} \alpha^{\lfloor k / 2\rfloor}$. Value $\alpha$ is set so that the minimum amplitude gain corresponds to the minimum amplitude in the collection of RPS test signals (see $\S 4.3 .4$ ), i.e. $\alpha \simeq 0.974$. This choice was empirically observed to optimize AS method.

Results Fig. 5 displays the separation error for AS and RPS methods. It shows that, for both methods, estimation of orders higher than 5 are unusable (due to a relative error exceeding $0 \mathrm{~dB}$ ). Furthermore, error does not monotonically increases with $n$ : even orders (6 and 8$)$ perform better than previous odd orders (respectively 5 and 7 ). This is due to the choice of an odd truncation order, i.e. $N=9$.

Fig. 5 also shows that AS method has lower error than RPS, with a difference between $\sim 6 \mathrm{~dB}$ (for order 1) and $\sim 3 \mathrm{~dB}$ (for order 9). If a smaller number $K$ of test signals is used for AS, or if gains $\alpha_{k}$ are not properly tuned, separation error drastically increases.

\subsection{Kernel identification evaluation}

Experiment description The same system and experiment as before is used. The error for kernel estimation is given by $\varepsilon_{n}=\operatorname{RMS}\left(\tilde{h}_{n}-h_{n}\right) / \operatorname{RMS}\left(h_{n}\right)$, where $\tilde{h}_{n}$ is the estimation of order $h_{n}$. Least-Squares identification methods are tested and compared, using either:

- method (A) on output $y$ corresponding to the reference signal;

- method (B) on orders $y_{n}$ estimated via AS;

- method (B) on orders $y_{n}$ estimated via RPS;

- method (C) on signals $y_{n, q}$ estimated via RPS, i.e. RPS without step (iv).

The direct approach relies only on one measurement of $L$ samples, and is therefore a far quicker experiment than using separation methods. For a meaningful comparison, a measurement of $K L$ samples should be used; but this would drastically increase the size of the 


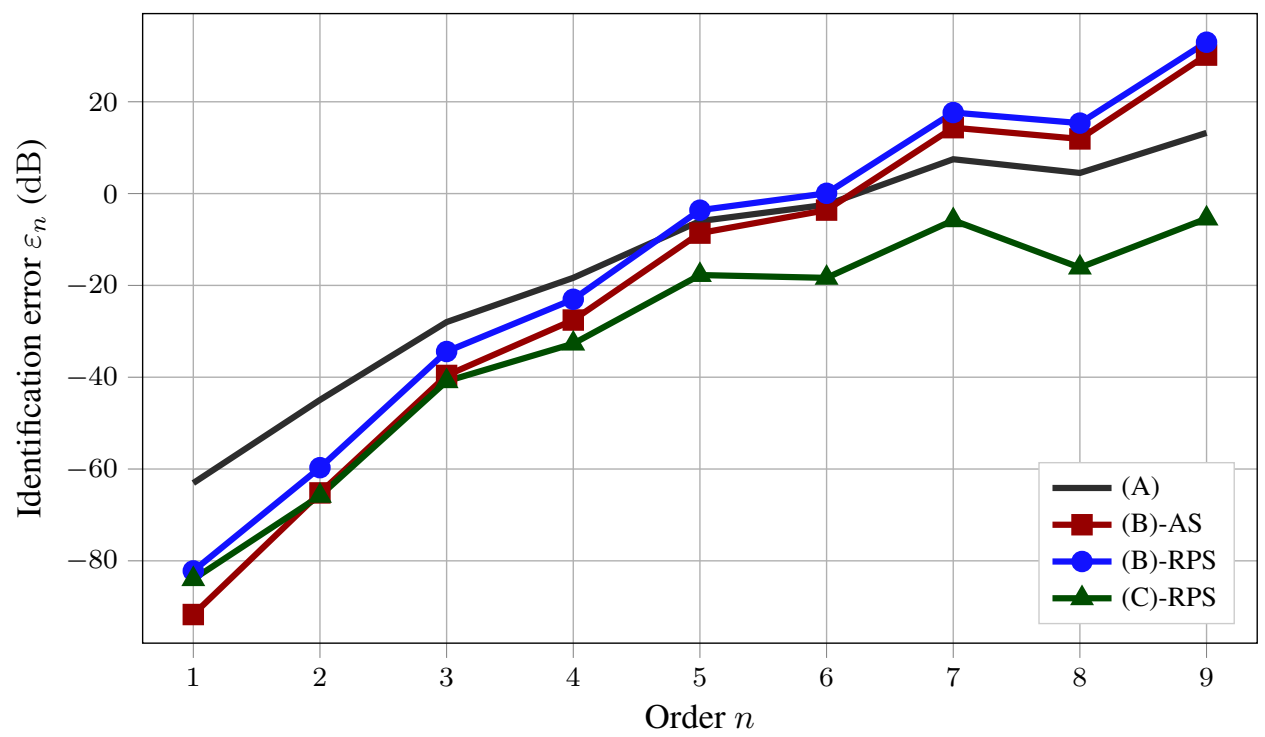

Figure 6. Comparison of identification error w.r.t order $n$ for LS identification using output $y$ ( $\longrightarrow$ ), orders $y_{n}$ estimated via AS method (- $)$, orders $y_{n}$ estimated via RPS method $(-\boldsymbol{c})$ and terms $y_{n, q}$ estimated via RPS method ( $\left.-\boldsymbol{C}\right)$. Median value for all experiments are represented.

combinatorial matrix $C$, and consequently the memory and CPU usage ${ }^{12}$. For application on real-life systems with longer memory $M$, this approach would lead to extremely large matrix sizes and intractable computation.

Results Estimation errors are shown in Fig. 6. As for the separation, the same non-monotonic behaviour is observed, which is still due to the choice of the truncation.

Direct identification (A) has an error similar to the SNR for order 1; when $n$ increases, this error grows rapidly. Previous observations on AS and RPS separation error still apply for identification error of both (B)-AS and (B)-RPS. In comparison to (A), those methods have lower error for kernels of orders 1 to 4 , but worse estimation for orders 7 to 9 .

Using interconjugate signals instead of homogeneous orders improves greatly the estimation of Volterra kernels. Method (C)-RPS is $\sim 20 \mathrm{~dB}$ lower than (A) for all orders. In comparison to (B)-RPS, this method ameliorates identification, especially for higher orders: this is the only method where identification results are lower than $0 \mathrm{~dB}$ for orders higher than 7 . This is explained by the fact that, for the same number of coefficients to estimate, more data is available when using signals $y_{n, q}$ (i.e. $(n+1) L$ instead of $L$ ).

\section{Conclusions and perspectives}

In this paper, the exploitation of phase deviation in Volterra series output for separating homogeneous orders has been studied. These results have then been used to devise new kernel identification methods in the Least-Squares formalism. Numerical experiments have been carried out to illustrate the amelioration brought in terms of estimation error.

The concept of interconjugate signals has been introduced. This notion permits, when working with a real-valued input, to consider the effect of the Volterra series on the complex-valued

\footnotetext{
${ }^{12}$ For example, in the limited case of this experiment, increasing the number of samples from $L$ to $K L$ would make the memory usage to store the matrix $\boldsymbol{C}$ alone going from $76 \mathrm{MiB}$ to $14.5 \mathrm{GiB}$.
} 
analytic signal. Using this concept, a new separation method has been proposed, relying on the 2-dimensional Discrete Fourier Transform. Furthermore, the use of interconjugate signals has been shown to improve kernel identification for higher orders.

The new separation method presented in this paper has been successfully applied to a reallife system (Bouvier, 2018; Lebrun, Bouvier, Hélie, \& Roze, 2018). Future work will focus on the use of this prior separation stage for identification of more structured systems, such as block-structured or polynomial nonlinear state-space systems.

\section{Appendix A. Proof of Theorem 4.4}

Consider the hypothesis of Theorem 4.4.

Because $2 \operatorname{Re}\left[\left(\theta_{1}+\theta_{2}\right) u\right]=\theta_{1} u+\overline{\theta_{1} u}+\theta_{2} u+\overline{\theta_{2} u}$, the $n$-linearity and symmetry of $\mathrm{V}_{n}$ gives the following expression for the output of order $n$ :

$$
y_{n}(t)=\sum_{\substack{k_{1}, k_{2}, k_{3}, k_{4}=0 \\
k_{1}+k_{2}+k_{3}+k_{4}=n}}^{n}\left(\begin{array}{c}
n \\
k_{1}, k_{2}, k_{3}, k_{4}
\end{array}\right) \theta_{1}^{k_{1}-k_{2}} \theta_{2}^{k_{3}-k_{4}} y_{n, k_{2}+k_{4}}(t) .
$$

Consider the change of variables

$$
\left\{\begin{array} { l } 
{ l _ { 1 } = k _ { 1 } + k _ { 2 } } \\
{ l _ { 2 } = k _ { 3 } + k _ { 4 } } \\
{ p _ { 1 } = k _ { 1 } - k _ { 2 } } \\
{ p _ { 2 } = k _ { 3 } - k _ { 4 } }
\end{array} \Leftrightarrow \left\{\begin{array}{l}
k_{1}=\left(l_{1}+p_{1}\right) / 2 \\
k_{2}=\left(l_{1}-p_{1}\right) / 2 \\
k_{3}=\left(l_{2}+p_{2}\right) / 2 \\
k_{4}=\left(l_{2}-p_{2}\right) / 2
\end{array}\right.\right.
$$

Then it is straightforward to show that the new variables $l_{i}, p_{i}, i=1,2$ respect the following conditions

$$
\begin{aligned}
& n=l_{1}+l_{2} \\
& p_{i} \equiv l_{i}[2] \quad \Rightarrow \quad p_{1}+p_{2} \equiv n[2] \\
& \left|p_{i}\right| \leq l_{i} \quad \Rightarrow \quad\left|p_{1}\right|+\left|p_{2}\right| \leq n
\end{aligned}
$$

Applying the change of variables (A2) to (A1) gives

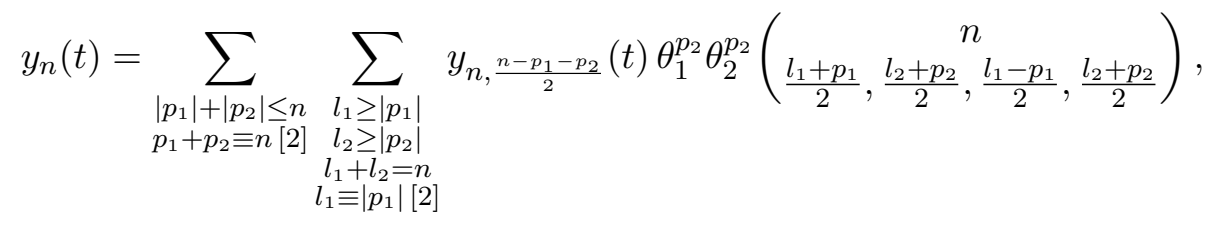

which can be simplified as

$$
y_{n}(t)=\sum_{\substack{\left|p_{1}\right|+\left|p_{2}\right| \leq n \\ p_{1}+p_{2} \equiv n[2]}} \theta_{1}^{p_{2}} \theta_{2}^{p_{2}} y_{n, \frac{n-p_{1}-p_{2}}{2}}(t) \sum_{\substack{l=\left|p_{1}\right| \\ l \equiv\left|p_{1}\right|[2]}}^{n-\left|p_{2}\right|}\left(\frac{l+p_{1}}{2}, \frac{n-l+p_{2}}{2}, \frac{l-p_{1}}{2}, \frac{n-l-p_{2}}{2}\right) \text {. }
$$


The second summation is equal to $\kappa_{\left|p_{1}\right|,\left|p_{2}\right|}^{(n)}$ as defined in (18). This is easily seen by observing that the multinomial coefficient is invariant under any sign change of $p_{1}$ and/or $p_{2}$. The total output is then

$$
y(t)=\sum_{n=0}^{N} \sum_{\substack{\left|p_{1}\right|+\left|p_{2}\right| \leq n \\ p_{1}+p_{2} \equiv n[2]}} \theta_{1}^{p_{2}} \theta_{2}^{p_{2}} \kappa_{\left|p_{1}\right|,\left|p_{2}\right|}^{(n)} y_{n, \frac{n-p_{1}-p_{2}}{2}}(t) .
$$

The two sums can be swapped as follows

$$
y(t)=\sum_{\left|p_{1}\right|+\left|p_{2}\right| \leq N} \theta_{1}^{p_{2}} \theta_{2}^{p_{2}} \sum_{\substack{n=\left|p_{1}\right|+\left|p_{2}\right| \\ n \equiv\left(p_{1}+p_{2}\right)[2]}}^{N} \kappa_{\left|p_{1}\right|,\left|p_{2}\right|}^{(n)} y_{n, \frac{n-p_{1}-p_{2}}{2}}(t),
$$

which concludes the proof.

\section{References}

Bard, D., \& Sandberg, G. (2007). Modeling of Nonlinearities in Electrodynamic Loudspeakers. In Audio engineering society convention 123.

Batselier, K., Chen, Z., \& Wong, N. (2017). Tensor Network alternating linear scheme for MIMO Volterra system identification. Automatica, 84, 26-35.

Birpoutsoukis, G., Csurcsia, P. Z., \& Schoukens, J. (2018). Efficient multidimensional regularization for Volterra series estimation. Mechanical Systems and Signal Processing, 104, 896-914.

Birpoutsoukis, G., Marconato, A., Lataire, J., \& Schoukens, J. (2017). Regularized nonparametric Volterra kernel estimation. Automatica, 82, 324-327.

Bouvier, D. (2018). Identification de systèmes non linéaires représentés en séries de volterra: applications aux systèmes sonores (Unpublished doctoral dissertation). Université Pierre et Marie Curie, Paris, France.

Bouvier, D., Hélie, T., \& Roze, D. (2017). Nonlinear Homogeneous Order Separation for Volterra Series Identification. In Proceedings of the 20th International Conference on Digital Audio Effects (DAFx'17) (pp. 3-10). Edinburgh, UK.

Bouvier, D., Hélie, T., \& Roze, D. (2018). Homophase signals separation for Volterra series identification. In Proceedings of the 57th IEEE Conference on Decision and Control (pp. 3854-3861).

Boyd, S., \& Chua, L. O. (1985). Fading memory and the problem of approximating nonlinear operators with Volterra series. IEEE Transactions on Circuits and Systems, 32(11), 1150-1161.

Boyd, S., Chua, L. O., \& Desoer, C. A. (1984). Analytical foundations of Volterra series. IMA Journal of Mathematical Control and Information, 1(3), 243-282.

Boyd, S., Tang, Y., \& Chua, L. O. (1983). Measuring Volterra kernels. IEEE Transactions on Circuits and Systems, 30(8), 571-577.

Brockett, R. W. (1977). Convergence of Volterra series on infinite intervals and bilinear approximations. In Nonlinear systems and applications (pp. 39-46). Elsevier.

da Rosa, A., Campello, R. J., \& Amaral, W. C. (2007). Choice of free parameters in expansions of discrete-time Volterra models using Kautz functions. Automatica, 43(6), 1084-1091.

Favier, G., Kibangou, A. Y., \& Bouilloc, T. (2012). Nonlinear system modeling and identification using Volterra-PARAFAC models. International Journal of Adaptive Control and Signal Processing, $26(1), 30-53$.

Franz, M. O., \& Schölkopf, B. (2006). A unifying view of Wiener and Volterra theory and polynomial kernel regression. Neural computation, 18(12), 3097-3118. 
Gardner, W. A., \& Archer, T. L. (1993). Exploitation of cyclostationarity for identifying the Volterra kernels of nonlinear systems. IEEE Transactions on Information Theory, 39(2), 535-542.

Golub, G. H., \& Van Loan, C. F. (2012). Matrix computations (Vol. 3). Johns Hopkins University Press.

Halme, A., Orava, J., \& Blomberg, H. (1971). Polynomial operators in non-linear systems theory. International Journal of Systems Science, 2(1), 25-47.

Hélie, T., \& Laroche, B. (2014). Computable convergence bounds of series expansions for infinite dimensional linear-analytic systems and application. Automatica, 50(9), 2334-2340.

Kibangou, A. Y., \& Favier, G. (2010). Identification of fifth-order volterra systems using iid inputs. IET Signal Processing, 4(1), 30-44.

Kibangou, A. Y., Favier, G., \& Hassani, M. M. (2005). Selection of generalized orthonormal bases for second-order Volterra filters. Signal Processing, 85(12), 2371-2385.

Lebrun, T., Bouvier, D., Hélie, T., \& Roze, D. (2018). Estimation de paramètres d'un hautparleur électrodynamique non linéaire. Société Française d'Acoustique. (14ème Congrès Français d'Acoustique)

Lee, G. (1997). Estimation of non-linear system parameters using higher-order frequency response functions. Mechanical Systems and Signal Processing, 11(2), 219-228.

Marmarelis, V. Z. (1993). Identification of nonlinear biological systems using Laguerre expansions of kernels. Annals of biomedical engineering, 21(6), 573-589.

Mathews, V. J., \& Sicuranza, G. L. (2000). Polynomial signal processing. John Wiley \& Sons, Inc.

Pan, V. Y. (2016). How bad are Vandermonde matrices? SIAM Journal on Matrix Analysis and Applications, 37(2), 676-694.

Rébillat, M., Hennequin, R., Corteel, É., \& Katz, B. F. G. (2011). Identification of cascade of Hammerstein models for the description of nonlinearities in vibrating devices. Journal of Sound and Vibration, 330(5), 1018-1038.

Rugh, W. J. (1981). Nonlinear system theory. Johns Hopkins University Press Baltimore.

Schoukens, M., \& Tiels, K. (2017). Identification of block-oriented nonlinear systems starting from linear approximations: A survey. Automatica, 85, 272-292.

Simpson, R. J., \& Power, H. M. (1972). Correlation techniques for the identification of non-linear systems. Measurement and Control, 5(8), 316-321.

Tiels, K., \& Schoukens, J. (2014). Wiener system identification with generalized orthonormal basis functions. Automatica, 50(12), 3147-3154.

Tseng, C.-H., \& Powers, E. J. (1995). Identification of cubic systems using higher order moments of iid signals. IEEE transactions on signal processing, 43(7), 1733-1735.

Volterra, V. (1887). Sopra le funzioni che dipendono da altre funzioni. Tipografia della Reale Accademia dei Lincei.

Wiener, N. (1942). Response of a nonlinear device to noise. Massachusetts Inst. Technology, Rept, 129.

Zhang, B., \& Billings, S. (2017). Volterra series truncation and kernel estimation of nonlinear systems in the frequency domain. Mechanical Systems and Signal Processing, 84, 39-57. 